Supporting Information

\title{
Alignment, Electronic Properties, Doping, and On-Chip Growth of Colloidal PbSe Nanowires
}

Dmitri V. Talapin, ${ }^{1,2 *}$ Charles T. Black, ${ }^{2}$ Cherie R. Kagan, ${ }^{2}$ Elena V. Shevchenko, ${ }^{1,2}$ Ali Afzali ${ }^{2}$, and Christopher B. Murray ${ }^{2}$

The Molecular Foundry, Lawrence Berkeley National Laboratory, Berkeley 94720, CA and IBM Research Division, T. J. Watson Research Center, 1101 Kitchawan Rd., Yorktown Heights, New York 10598, USA

*Corresponding author: dvtalapin @lbl.gov (D.V.T.)

${ }^{1}$ The Molecular Foundry, LBNL

${ }^{2}$ IBM T. J. Watson Research Center 


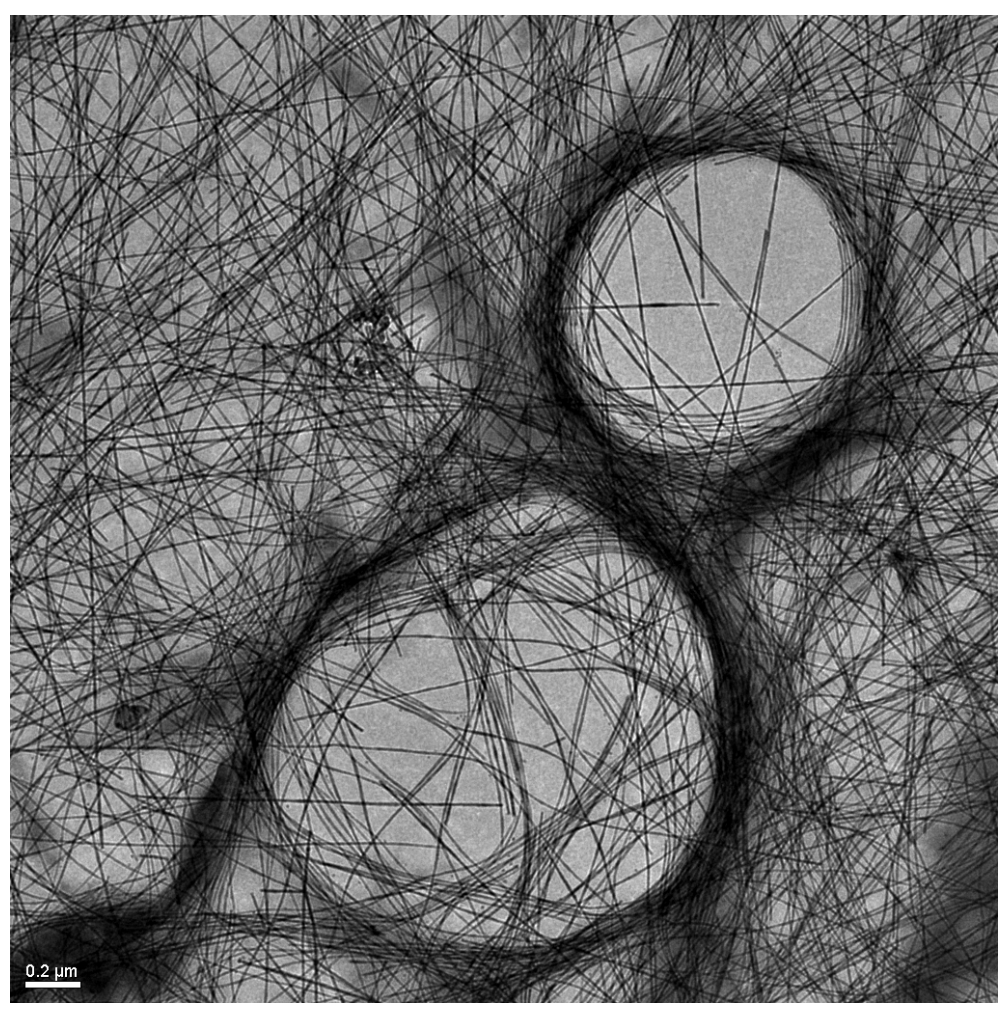

Figure S1. TEM image of straight, single-crystalline PbSe nanowires synthesized in a colloidal solution. 


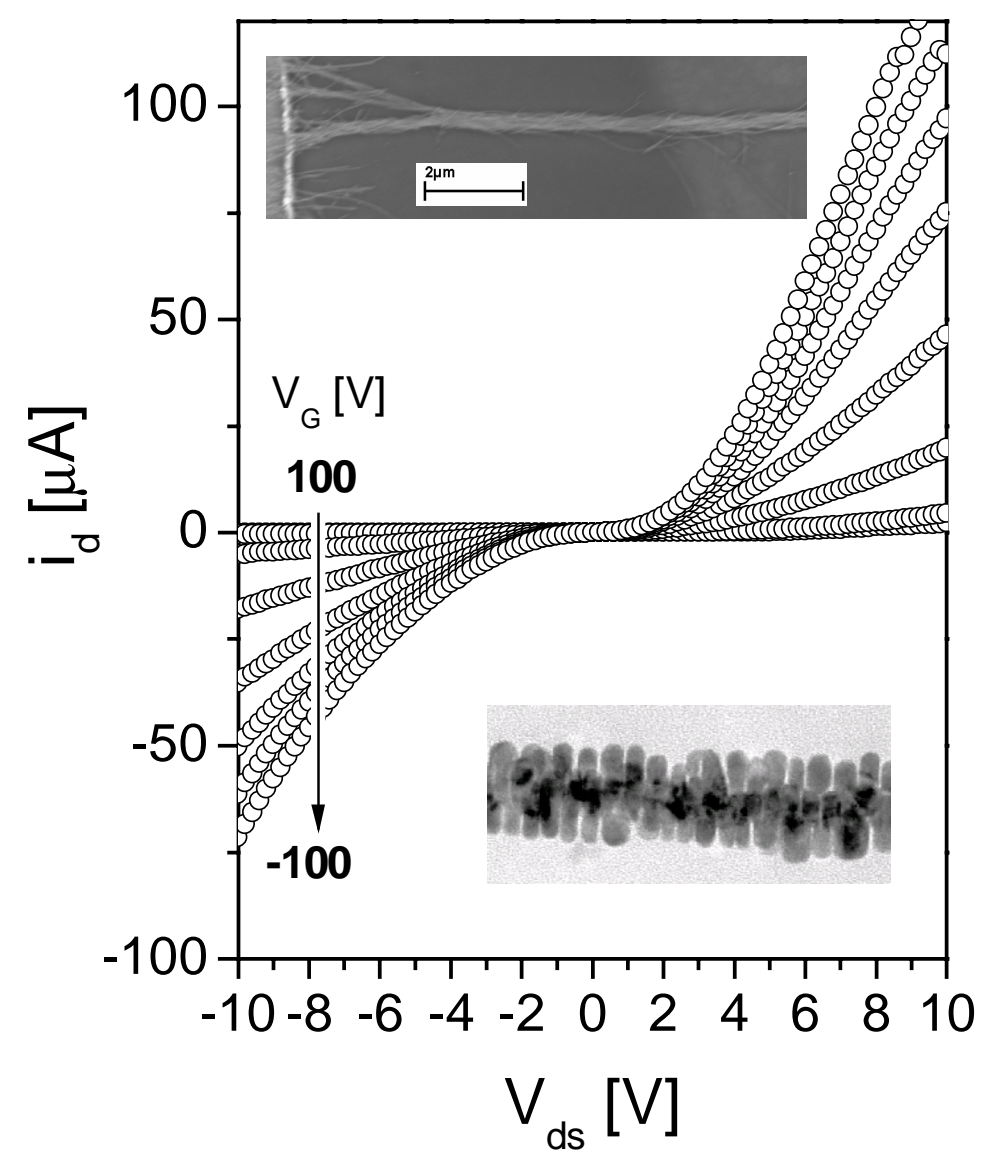

Figure S2. Transport characteristics of branched PbSe nanowires assembled into "nano-ropes". Plot of drain current $I_{\mathrm{d}}$ versus drain-source voltage $V_{\mathrm{ds}}$, as a function of gate voltage $V_{\mathrm{g}}$ for assynthesized p-type branched PbSe nanowires. The channel length is $20 \mu \mathrm{m}$. Insets show SEM image of a "nano-rope" (top) and TEM image of individual branched $\mathrm{PbSe}$ nanowire (bottom). 


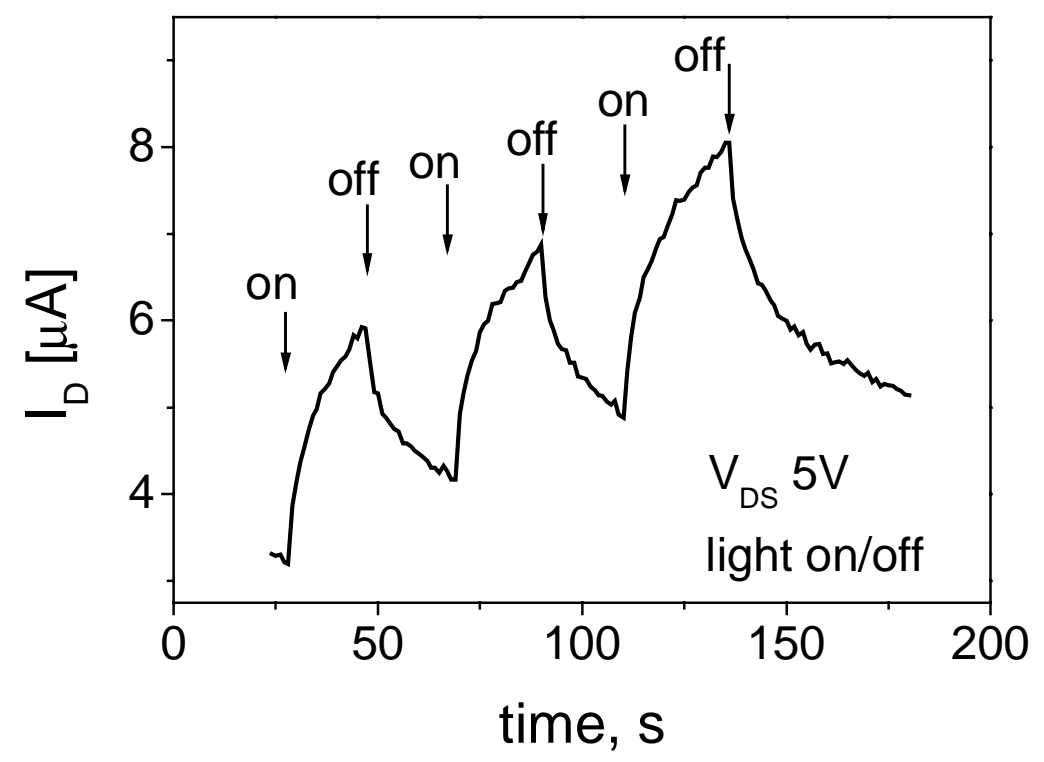

Figure S3. The modulation of drain current by illuminating on-chip grown $\mathrm{PbSe}$ nanowires with visible light. 